\title{
Impact of electron-vibron interaction on the bound states in the continuum
}

\author{
C. Álvarez ${ }^{1}$, F. Domínguez-Adame ${ }^{1,2}$, P. A. Orellana ${ }^{3}$ and E. Díaz ${ }^{1}$ \\ 1 GISC, Departamento de Física de Materiales, Universidad Complutense, E-28040 \\ Madrid, Spain \\ 2 Department of Physics, University of Warwick, Coventry, CV4 7AL, United Kingdom \\ 3 Departamento de Física, Universidad Técnica Federico Santa María, Casilla 110 V, \\ Valparaíso, Chile
}

\begin{abstract}
We investigate the nonequilibrium transport properties of a coupled quantum dot system connected in parallel to two leads, including electron-vibron interaction. It is known that in the absence of interaction the system supports a bound state in the continuum. This state is revealed as a Fano antiresonance in the transmission when the energy levels of the dots are detuned. Using the Keldysh nonequilibrium Green's function formalism, we find that the occurrence of the Fano antiresonance arises even if the electron-vibration interaction is taken into account. We also examine the impact of the coupling to the leads in the linear response of the system. We conclude that the existence of bound states in the continuum in coupled quantum dot systems is a robust phenomenom, opening the possibility of its observation in experiments.
\end{abstract}

Keywords: Bound states, quantum dots, electron-vibron coupling PACS: 73.63.Kv, 72.10.Di, 73.63.-b

\section{Introduction}

Double quantum dot (DQD) systems are artificial ultra small structures that share electronic properties with diatomic molecules $[1,2]$. Due to the advances in fabrication techniques, many aspects of the physics involved at the quantum level of few-electron systems can by now be tested using DQDs. For this reason, DQDs are emerging as versatile systems to explore a variety 
of fundamental physics phenomena, such as Kondo states [3, 4, 5, 6], quantum interference [7, 8], Coulomb blockade [9, 10, 11], Fano effect $[12,13,14]$ and correlation-induced resonances $[15,16]$.

The similarity of artificial nanostructures, like semiconductor superlattices and quantum dots, and atomic systems paved the way to experimentally validate several theoretical predictions formulated much earlier than the advent of nanotechnology. A remarkable example is the occurrence of bound states in the continuum (BICs). Shortly after the formulation of quantum mechanics, in 1929 von Neumann and Wigner constructed a spatially oscillating attractive potential that supported a bound state (square integrable) above the potential barrier [17]. These exotic states were regarded as a mere theoretical curiosity until 1992, when Capasso et al. measured the absorption spectrum at low temperature of a GaInAs quantum well with Bragg reflector barriers produced by a AlInAs/GaInAs superlattice [18]. A well defined line at $360 \mathrm{meV}$ in the spectrum was attributed to electron excitations from the ground state of the quantum well to a localized level well above the AlInAs band edge. Nevertheless, Plotnik et al. claimed that this state is not a true BIC but a defect mode residing in the gap [19]. More recently, Albo et al. characterized III-V-N (diluted nitrides) quantum wells by intersubband photocurrent spectroscopy. They stated the these samples exhibit signatures of BICs resulting from the hybridization of nitrogen-related defect states and the extended states of the conduction band [20].

Aiming to introduce a physical realizable system to reveal the existence of BICs in transport experiments, we have recently studied a parallel DQD system [21]. The coupling between the BIC and the continuum energy states was controlled by detuning the energy levels of each quantum dot using gate voltages. We showed that the transmission probability displays a Fano antiresonance profile [22] at the energy of the BIC. More important, when the gate voltages are modulated harmonically in time, the energy of the resonance can be continuously shifted by varying the driving frequency. We predicted that the conductance at low temperature presents a minimum when the BIC crosses the Fermi level by varying the driving frequency. The notion of BICs sustained by systems subjected to time periodic fields has been further investigated in Refs. [23] and [24]. However, it is still an open question to what extend interactions would mask the effect in a real experiment. Žtiko et al. have shown that the so-called dark states in parallel DQD systems, corresponding to BICs discussed in Ref. [21], are robust against electronelectron interactions, at least in the Kondo regime [25]. 
In this work we study the impact of electron-vibron interaction on transport properties when the system supports a BIC, using the Keldysh nonequilibrium Green's function formalism [26]. Specifically, we consider a DQD connected in parallel to two leads and assume that the electron interact with a vibrational degree of freedom localized at each dot. We examine the spectral function, the electric current through the DQD system and the differential conductance. We study different configurations of the energy levels of the dots as well as the role of the contacts between the dots and the leads. When the symmetry of the system is broken (different energy levels of the dots or asymmetric couplings to the leads), the spectral density and the differential conductance reveal the occurrence of a BIC in the energy spectrum. This result is not substantially affected by the electron-vibron coupling and we conclude that transport experiments in DQD systems could uncover these exotic states.

\section{Model}

We consider two quantum dots forming a DQD system, connected to left and right leads by tunnel couplings, as shown schematically in figure 1 . The bias voltage between the leads, $V$, is given by $e V=\mu_{L}-\mu_{R}$, where $\mu_{\alpha}$ is the chemical potential of the lead $\alpha=L, R$. Only one energy level in each dot is assumed relevant and electron-electron interaction is neglected. The energy of the local level can be tuned by gate voltages, $V_{g 1}$ and $V_{g 2}$, depicted in figure 1. In addition, the dots are far apart and direct tunneling between the dots is suppressed. The electron interacts with a local vibration mode at each dot and, for simplicity, we assume the same frequency $\omega_{0}$ for both modes.

The Hamiltonian describing the whole system can be written as $H=$ $H_{0}+H_{\mathrm{e}-\text { leads }}+H_{\mathrm{e}-\mathrm{vib}}$. Here $H_{0}$ describes the dynamics of the noninteracting system (we set $\hbar=1$ hereafter)

$$
H_{0}=\sum_{i}\left(\varepsilon_{i} d_{i}^{\dagger} d_{i}+\omega_{0} a_{i}^{\dagger} a_{i}\right)+\sum_{k, \alpha} \varepsilon_{\alpha, k} c_{\alpha, k}^{\dagger} c_{\alpha, k}
$$

where the index $i=1,2$ runs over the quantum dots and $\alpha=L, R$ indicates

the lead. Here $c_{\alpha, k}^{\dagger}\left(c_{\alpha, k}\right)$ denotes the creation (annihilation) operator of a conduction electron in the semi-infinite lead $\alpha$ with crystal momentum $k$ and energy $\varepsilon_{\alpha, k}$. Similarly, $d_{i}^{\dagger}\left(d_{i}\right)$ is the creation (annihilation) operator of 


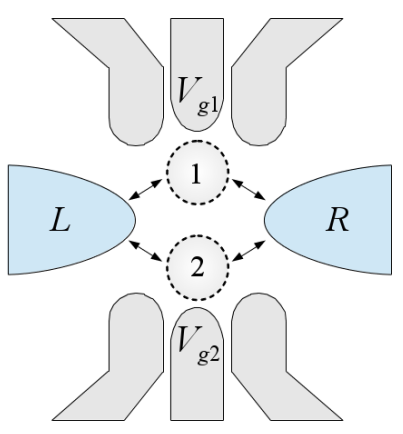

Figure 1: Schematic diagram of the DQD system coupled in parallel to left and right leads. Two gate voltages, $V_{g 1}$ and $V_{g 2}$, control the energy of the local level of each dot.

an electron in the dot $i$ with energy $\varepsilon_{i}$. Finally, the creation (annihilation) operator of a local vibration mode in the dot $i$ with frequency $\omega_{0}$ is denoted by $a_{i}^{\dagger}\left(a_{i}\right)$.

The dots are tunnel coupled to both leads, as shown schematically in figure 1. Therefore, the corresponding Hamiltonian reads

$$
H_{\mathrm{e}-\text { leads }}=\sum_{i, k, \alpha} V_{i, k, \alpha} c_{\alpha, k}^{\dagger} d_{i}+\text { H.c. }
$$

where H.c. stands for Hermitian conjugate. We assume a Holstein-type coupling between the electron and the vibron [27]

$$
H_{\mathrm{e}-\mathrm{ph}}=\lambda \sum_{i}\left(a_{i}^{\dagger}+a_{i}\right) d_{i}^{\dagger} d_{i} .
$$

For simplicity we take the same coupling constant $\lambda$ in both dots.

We now apply the Lang-Firsov nonperturbative canonical transformation $\widetilde{H}=e^{S} H e^{-S}$, where the operator $S$ is defined as $S=\left(\lambda / \omega_{0}\right) \sum_{i}\left(a_{i}^{\dagger}-\right.$ $\left.a_{i}\right) d_{i}^{\dagger} d_{i}[28]$. The transformed Hamiltonian takes the form $\widetilde{H}=\widetilde{H}_{0}+\widetilde{H}_{\text {int }}$, where the transformed noninteracting term is given as

$$
\widetilde{H}_{0}=\sum_{i} \widetilde{\varepsilon}_{i} d_{i}^{\dagger} d_{i}+\sum_{k, \alpha} \varepsilon_{\alpha, k} c_{\alpha, k}^{\dagger} c_{\alpha, k},
$$

where $\widetilde{\varepsilon}_{i}=\varepsilon_{i}-g \omega_{0}$ and $g=\lambda^{2} / \omega_{0}^{2}$ is the Huang-Rhys factor. The resulting interaction Hamiltonian $\widetilde{H}_{\text {int }}$ is similar to $H_{\mathrm{e} \text {-leads }}$ given in Eq. (2), replacing $V_{i, k, \alpha}$ by $V_{i, k, \alpha} X$ with $X=\exp \left[\left(\lambda / \omega_{0}\right)\left(a_{i}^{\dagger}-a_{i}\right)\right]$. The canonical transformation is exact but it does not diagonalize the Hamiltonian. In other words, 
$\widetilde{H}_{\text {int }}$ contains products of boson and fermion operators. Since we are dealing with localized vibration modes, it is reasonable to replace the operator $X$ by its expectation value $\langle X\rangle=\exp [-\xi(T) / 2]$, where $\xi(T)=g\left(2 N_{\text {vib }}+1\right)$, $N_{\text {vib }}=1 /\left[\exp \left(\beta \omega_{0}\right)-1\right]$ and $\beta=1 / k_{B} T[29,30]$. Defining $\widetilde{V}_{i, k, \alpha}=V_{i, k, \alpha}\langle X\rangle$, the transformed interaction Hamiltonian is approximately taken as

$$
\widetilde{H}_{\text {int }}=\sum_{i, k, \alpha} \widetilde{V}_{i, k, \alpha} c_{\alpha, k}^{\dagger} d_{i}+\text { H.c. }
$$

This approach is valid when the coupling between the DQD system and the leads is weaker than the electron-vibron coupling, namely $\left|\widetilde{V}_{i, k, \alpha}\right| \ll \lambda$.

\section{Spectral function and differential conductance}

Nonequilibrium transport properties of an interaction region coupled to two leads can be obtained with the help of the Keldysh Green's function technique [31]. The magnitudes of interest are the spectral matrix

$$
\mathcal{A}(\omega)=\mathrm{i}\left[G^{\mathrm{r}}(\omega)-G^{\mathrm{a}}(\omega)\right],
$$

along with the spectral function $A(\omega)=\operatorname{Tr}[\mathcal{A}(\omega)] / 2$, the transmission coefficient

$$
T(\omega)=\operatorname{Tr}\left[G^{\mathrm{a}}(\omega) \Gamma^{R} G^{\mathrm{r}}(\omega) \Gamma^{L}(\omega)\right],
$$

and the electric current

$$
I=\frac{e}{2 h} \int d \omega \operatorname{Tr}\left\{\left[f_{L}(\omega) \Gamma^{L}-f_{R}(\omega) \Gamma^{R}\right] \mathcal{A}(\omega)+\mathrm{i}\left(\Gamma^{L}-\Gamma^{R}\right) G^{<}(\omega)\right\},
$$

where $f_{\alpha}(\omega)=1 /\left\{\exp \left[\beta\left(\omega-\mu_{\alpha}\right)\right]+1\right\}^{-1}$ is the Fermi-Dirac distribution function of the lead $\alpha$. The tunnel coupling of the DQD system to both leads is encoded in the matrices [32]

$$
\Gamma^{L}=\Gamma_{0}\left(\begin{array}{cc}
1 & \sqrt{a} \\
\sqrt{a} & a
\end{array}\right), \quad \Gamma^{R}=\Gamma_{0}\left(\begin{array}{cc}
a & \sqrt{a} \\
\sqrt{a} & 1
\end{array}\right) .
$$

Here $\Gamma_{0}$ and $a$ are phenomenological parameters describing the different coupling of each dot to both leads. This coupling corresponds to the configuration studied in Ref. [12]. Once the current is computed, one can obtain the dimensionless differential conductance as $\mathcal{G}=(d I / d V) / G_{0}$, where $G_{0}=2 e^{2} / h$ is the quantum of conductance. 
The various Green's functions in the above expressions have the usual meaning in the nonequilibrium Keldysh formalism [26]. They can be calculated using standard techniques and for brevity we only quote the main results [30, 32]. The lesser and greater Green's functions can be expressed as

$$
\begin{aligned}
& G^{<}(\omega)=\sum_{n=-\infty}^{\infty} L_{n} \widetilde{G}^{<}\left(\omega+n \omega_{0}\right), \\
& G^{>}(\omega)=\sum_{n=-\infty}^{\infty} L_{n} \widetilde{G}^{>}\left(\omega-n \omega_{0}\right),
\end{aligned}
$$

where at finite temperature

$$
L_{n}=e^{-\xi(T)+n \beta \omega_{0} / 2} I_{n}\left(\sqrt{\xi^{2}(T)-g^{2}}\right),
$$

$I_{n}(z)$ being the modified Bessel function of integer order[33], and at zero temperature

$$
L_{n}= \begin{cases}e^{-\xi(0)} g^{n} / n ! & \text { if } n \geq 0 \\ 0 & \text { if } n<0\end{cases}
$$

The dressed lesser and greater Green's functions can be calculated from the Keldysh equation $\widetilde{G}^{<(>)}(\omega)=\widetilde{G}^{\mathrm{r}}(\omega) \widetilde{\Sigma}^{<(>)}(\omega) \widetilde{G}^{\mathrm{a}}(\omega)$, where the self-energies are given by

$$
\begin{aligned}
& \widetilde{\Sigma}^{<}(\omega)=\mathrm{i} e^{\xi(T)}\left[f_{L}^{(e)}(\omega) \Gamma^{L}+f_{R}^{(e)}(\omega) \Gamma^{R}\right], \\
& \widetilde{\Sigma}^{>}(\omega)=-\mathrm{i} e^{\xi(T)}\left[f_{L}^{(h)}(\omega) \Gamma^{L}+f_{R}^{(h)}(\omega) \Gamma^{R}\right],
\end{aligned}
$$

with $f_{\alpha}^{(e)}(\omega)=f_{\alpha}(\omega)$ and $f_{\alpha}^{(h)}(\omega)=1-f_{\alpha}(\omega)$. The dressed retarded and advanced Green's functions are obtained as

$$
\begin{aligned}
& \widetilde{G}^{\mathrm{r}}(\omega)=\frac{1}{P(\omega)}\left(\begin{array}{cc}
\omega-\widetilde{\varepsilon}_{2}-\widetilde{\Sigma}_{22} & \widetilde{\Sigma}_{12} \\
\widetilde{\Sigma}_{21} & \omega-\widetilde{\varepsilon}_{1}-\widetilde{\Sigma}_{11}
\end{array}\right), \\
& \widetilde{G}^{\mathrm{a}}(\omega)=\widetilde{G}^{\mathrm{r}}(\omega)+\widetilde{G}^{<}(\omega)-\widetilde{G}^{>}(\omega) .
\end{aligned}
$$

with $P(\omega)=\left(\omega-\widetilde{\varepsilon}_{2}-\widetilde{\Sigma}_{22}\right)\left(\omega-\widetilde{\varepsilon}_{1}-\widetilde{\Sigma}_{11}\right)-\widetilde{\Sigma}_{12} \widetilde{\Sigma}_{21}$ and $\widetilde{\Sigma}=(\mathrm{i} / 2) \exp [-\xi(T)]\left(\Gamma^{L}+\right.$ $\left.\Gamma^{R}\right)$. Finally, the retarded and advanced Keldysh Green's functions can be 
calculated from

$$
\begin{aligned}
G^{r}(\omega) & =\sum_{n=-\infty}^{\infty} L_{n}\left\{\widetilde{G}^{\mathrm{r}}\left(\omega-n \omega_{0}\right)-\frac{1}{2} \widetilde{G}^{<}\left(\omega+n \omega_{0}\right)\right. \\
& \left.+\frac{1}{2} \widetilde{G}^{<}\left(\omega-n \omega_{0}\right)\right\}, \\
G^{a}(\omega) & =\sum_{n=-\infty}^{\infty} L_{n}\left\{\widetilde{G}^{\mathrm{r}}\left(\omega-n \omega_{0}\right)+\frac{1}{2} \widetilde{G}^{<}\left(\omega+n \omega_{0}\right)\right. \\
& \left.+\frac{1}{2} \widetilde{G}^{<}\left(\omega-n \omega_{0}\right)-\widetilde{G}^{>}\left(\omega-n \omega_{0}\right)\right\} .
\end{aligned}
$$

Once the various Green's functions are calculated, the magnitudes of interest (6) can be readily obtained.

\section{Results}

The main emphasis of our analysis is to evaluate the impact of the electron-vibron coupling on the transport properties of the DQD system and the role of the leads. To avoid the profusion of free parameters, we set $\mu_{L}=0$ hereafter. We take $\omega_{0}$ as the unit of energy in our numerical calculations.

\subsection{Non-interacting DQD system}

To gain insight into the occurrence of BICs in the DQD system, we consider the non-interacting case by setting $\lambda=0$ for the moment. In this subsection we take $\varepsilon_{1}=-\varepsilon_{2} \equiv \varepsilon$ to obtain simpler expressions, although more general situations can be handled in the same way. A lengthy but straightforward calculation yields the following expression for the transmission coefficient $(6 \mathrm{~b})$

$$
T(\omega)=\frac{4 a \Gamma_{0}^{2} \omega^{2}}{D(\omega)},
$$

with

$$
D(\omega)=\left(\omega^{2}-\varepsilon^{2}\right)^{2}+\frac{\Gamma_{0}^{2}}{2}\left[\omega^{2}\left(1+6 a+a^{2}\right)+\varepsilon^{2}(1-a)^{2}\right]+\left[\frac{\Gamma_{0}}{2}(1-a)\right]^{4}
$$

The transmission coefficient vanishes at $\omega=0$ except if $\varepsilon=0$ and the

coupling to the leads is symmetric $(a=1)$. In this particular case the 
transmission coefficient presents a Lorentzian shape

$$
T(\omega)=\frac{4 \Gamma_{0}^{2}}{\omega^{2}+4 \Gamma_{0}^{2}}
$$

of width $2 \Gamma_{0}$. Therefore, unless the system is finely tuned $(\varepsilon=0$ and $a=1)$, in general the transmission coefficient shows a marked dip at $\omega=0$. For instance, when the coupling to the leads is symmetric $(a=1)$ but the energy levels of the dots are detuned $(\varepsilon \neq 0)$ one obtains

$$
T(\omega)=\frac{\omega^{2}}{\omega^{2}+\varepsilon^{4} / 4 \Gamma_{0}^{2}}
$$

for $|\omega|<\varepsilon$. We notice that the transmission coefficient displays a Fano antiresonance profile of width $\varepsilon^{2} / 2 \Gamma_{0}$. Similarly, when the energy levels are the same $(\varepsilon=0)$ but the coupling to the leads is asymmetric $(a \neq 1)$, the transmission shows again a Fano antiresonance around $\omega=0$, namely $T(\omega) \sim$ $\omega^{2} /\left(\omega^{2}+\gamma^{2}\right)$ where now the width is given by $\gamma=\Gamma_{0}(1-a)^{2} / \sqrt{8\left(1+6 a+a^{2}\right)}$.

The occurrence of a Fano antiresonance in the transmission also reflects itself in the differential conductance. Figure 2 shows the results for tuned levels $(\varepsilon=0)$ and symmetric or asymmetric couplings to the leads. If this coupling is exactly the same for both quantum dots $(a=1)$, the differential conductance displays a single peak around $e V=0$. However, in a more general situation when the coupling is not the same $(a \neq 1)$, the differential conductance displays a well defined dip at $\mathrm{eV}=0$, in perfect agreement with the Fano antiresonance observed in the transmission coefficient.

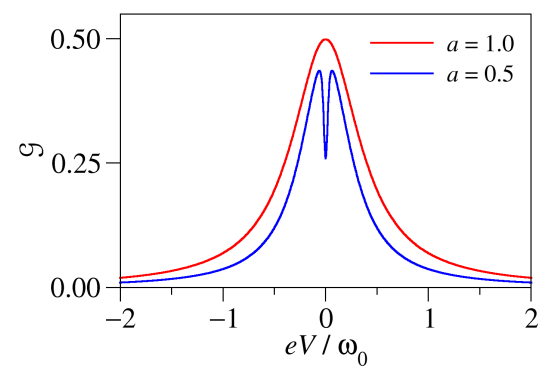

Figure 2: Dimensionless differential conductance for symmetric ( $a=1$, red solid line) and asymmetric $\left(a=0.5\right.$, blue solid line) couplings to the leads, at $k_{B} T=0.01$ and in absence of electron-vibron coupling $(\lambda=0)$. The energy levels of the dots are the same $(\varepsilon=0)$ and $\Gamma_{0}=0.2$. 
In the non-interacting DQD system, the spectral function $A(\omega)$ can also be calculated analytically

$$
A(\omega)=\frac{(1+a) \Gamma_{0}}{D(\omega)}\left[\omega^{2}+\varepsilon^{2}+\frac{1}{4}(1-a)^{2} \Gamma_{0}^{2}\right],
$$

where $D(\omega)$ is given in $(12 \mathrm{~b})$. It is then found that $A(\omega)$ presents Lorentzian profiles close to $\omega=0$ in all cases. For instance, when $\varepsilon=0$ and $a \neq 1$ one easily gets $A(\omega) \sim \gamma /\left(\omega^{2}+\gamma^{2}\right)$ if $|\omega|<\Gamma_{0}$, where $\gamma$ was defined above. More interesting results arise when the coupling to the leads is the same for both quantum dots $(a=1)$. Taking $|\omega|<\varepsilon<\Gamma_{0}$, the spectral function is approximately given as

$$
A(\omega) \simeq \frac{2 \Gamma_{0}}{\omega^{2}+4 \Gamma_{0}^{2}}+\frac{\varepsilon^{2} / 2 \Gamma_{0}}{\omega^{2}+\left(\varepsilon^{2} / 2 \Gamma_{0}\right)^{2}} .
$$

The spectral function is the sum of two Lorentzian profiles, originated from the superposition of two states. One of these states is strongly coupled to the continuum, giving rise a wide peak of width $2 \Gamma_{0}$. However, the other state is only weakly coupled to the continuum since the corresponding level broadening $\varepsilon^{2} / 2 \Gamma_{0}$ is small when $\varepsilon<\Gamma_{0}$. When the energy levels of the quantum dots are the same $(\varepsilon \rightarrow 0)$, the spectral function becomes

$$
A(\omega) \simeq \frac{2 \Gamma_{0}}{\omega^{2}+4 \Gamma_{0}^{2}}+\pi \delta(\omega)
$$

In this case the spectral function approaches a $\delta$-function, indicating the existence of a truly bound state with energy $\omega=0$ located at both quantum dots [21]. In other words, the localizd state becomes effectively decoupled from the continuum states but its energy lies within the band (BIC).

To conclude the analysis of the non-interacting DQD system, let us stress that the system supports BICs that can be detected by studying its transport properties. The transmission coefficient generally presents a Fano antiresonance profile except if the parameters are finely tuned, namely when $\varepsilon=0$ and $a=1$ it displays a Lorentzian shape. Correspondingly, the differential conductance shows a marked dip when the Fermi level matches the energy of the BIC.

\subsection{Interacting $D Q D$ system}

We now turn to our main goal, the effects of the electron-vibron coupling on the BICs discussed in the previous section. We apply the methodology 
presented in Sec. 2 to symmetric and asymmetric configurations of the DQD system when $\lambda$ is finite. For concreteness we take $\lambda=0.5$ in the numerical calculations.

Inelastic scattering events due to excitation and de-excitation of phonons are revealed as side peaks in both the transmission coefficient and the spectral function. Figure 3 shows the results for different configurations of DQD systems. In all cases the transmission coefficient (solid blue lines) and the spectral function (dashed red lines) present the zero-phonon line and side peaks due to emission or absorption of phonons. In the perfectly symmetric configuration ( $a=1$ and $\varepsilon_{1}=\varepsilon_{2}$ ), both the transmission coefficient and the spectral function are similar, as seen in figure 3(a). These magnitudes present a Lorentzian zero-phonon line whose width is roughly $2 \Gamma_{0}$, as in the noninteracting case given by equation (13b). According to equation (13c), in the absence of electron-vibron interaction the spectral density displays an aditional $\delta$-function peak when $\left|\varepsilon_{1}-\varepsilon_{2}\right|$ goes to zero. This singularity can not be observed in figure 3(a) due to the finite resolution of the numerics, as expected. However, a small imaginary part added to the energy of the quantum dots widens the BIC, which can be now detected as a narrow peak in the spectral density (see inset of figure 3). This leads to the conclusion that the electron-vibron interaction does not mask the main features of the BICs in the spectral density.

When the DQD is asymmetric $(a \neq 1$ and/or different quantum dot energies) the transmission coefficient vanishes at $\omega=\left(\widetilde{\varepsilon}_{1}+\widetilde{\varepsilon}_{2}\right) / 2=\left(\varepsilon_{1}+\right.$ $\left.\varepsilon_{2}\right) / 2-\lambda^{2} / \omega_{0}$. If only the couplings to the leads are different but the energy levels of the quantum dots are the same, both the transmission coefficient and the spectral function present similar trends, except for the vanishing of the latter at $\omega=0$, as seen in figure 3(b). On the contrary, if the energy levels of the quantum dots are different (lower panels of figure 3 ), the spectral function vanishes at $\omega=-\lambda^{2} / \omega_{0}$ while the transmission coefficient is maximum. This behaviour is found to be independent of the symmetry of the coupling to the leads.

Further insight into the behaviour of the BICs is obtained from the differential conductance. Figure 4 shows the results for different configurations of the DQD system. We observe the occurrence of the expected side-bands discussed above and a small energy shift $\lambda^{2} / \omega_{0}=0.25$ due the renormalization of the electron energy. Close to the (renormalized) band centre the dimensionless differential conductance presents a marked dip except if the system is perfectly symmetric $(a=1$ and $\varepsilon=0)$, as seen in figure 4(a). This behaviour 


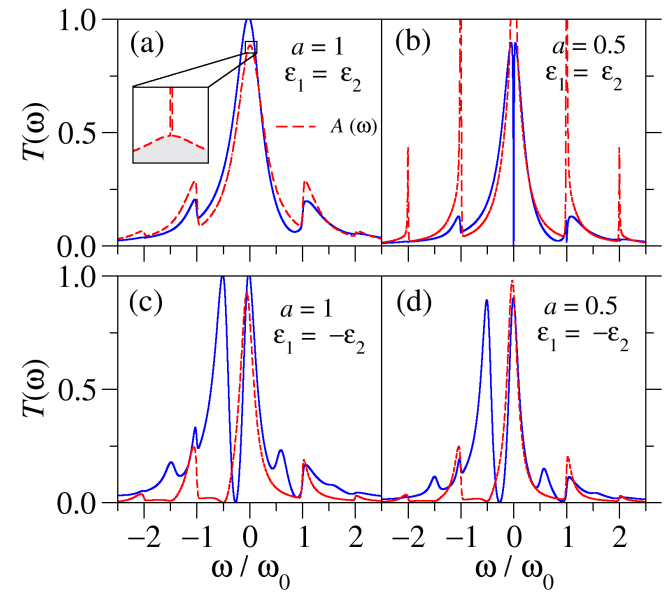

Figure 3: Transmission coefficient (solid blue lines) and spectral function in arbitrary units (dashed red lines) for symmetrically (left panels) and asymmetrically (right panels) connected DQD systems. System parameters are $\Gamma_{0}=0.2, \lambda=0.5, k_{B} T=0.01$ and $\omega_{0}=1$. In all cases $\varepsilon_{1}=\left|\varepsilon_{2}\right|=\lambda^{2} / \omega_{0}$. The inset shows an enlarged view of the spectral density when a small imaginary part is added to the energy of the quantum dots.

is in perfect correspondence to what we found in noninteracting systems (see figure 2) The dimensionless differential conductance exactly vanishes at the renormalized band centre when the quantum dots are detuned (see lower panels of figure 4). We have found numerically that the dip cannot be resolved above a threshold temperature of the order of $T_{\text {th }} \simeq 0.06 \omega_{0} / k_{B}$ with our chosen parameters. Taking $\omega_{0}=5-50 \mathrm{meV}$ as typical values of phonon energy in these DQD systems [34], the threshold temperature is found to be $T_{\mathrm{th}}=0.6-6 \mathrm{~K}$. Therefore, the signatures of BICs in the differential conductance should be observable even at temperatures slightly above the liquid helium temperature.

In our study we have assumed that interdot tunneling is negligible. Therefore, tunneling from one dot to the other is always an indirect process since it can only take place through the leads. In the literature, however, direct interdot tunneling is often taken into account. This amounts to include the term $t\left(d_{1}^{\dagger} d_{2}+d_{2}^{\dagger} d_{1}\right)$ in the Hamiltonian (1), where $t$ is the interdot hopping energy [32]. Dashed lines in figures 4(b) and 4(d) show the dimensionless differential conductance when the interdot tunneling is not negligible and the hopping energy is $t=0.4$. Notice that this hopping energy is also renormalized after the Lang-Firsov transformation, $\tilde{t}=t \exp [-\xi(T)]$. The rest 


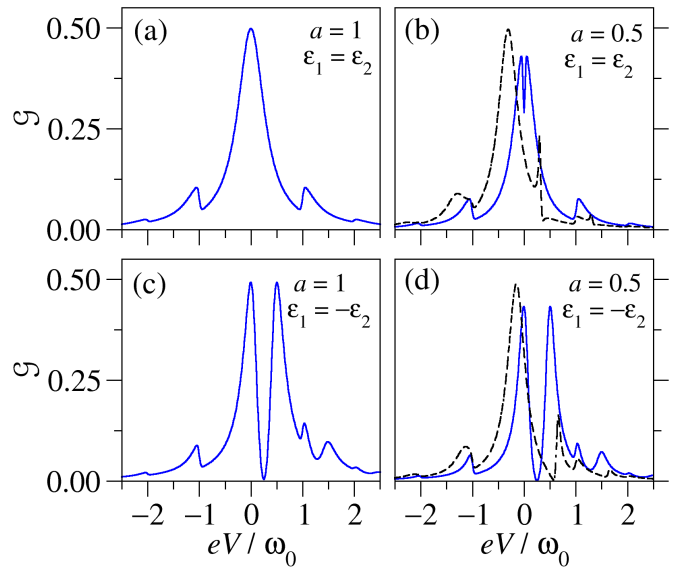

Figure 4: Dimensionless differential conductance for symmetrically (left panels) and asymmetrically (right panels) connected DQD systems. System parameters are $\varepsilon_{1}=\left|\varepsilon_{2}\right|=0.25$, $\Gamma_{0}=0.2, \lambda=0.5, k_{B} T=0.01$ and $\omega_{0}=1$. Dashed lines of the right panels shows the results when the interdot tunneling is not negligible (hopping energy is $t=0.4$ ). The rest of parameters are indicated in the legends.

of parameters correspond to symmetric coupling to the leads $(a=1)$ and $\varepsilon_{1}= \pm \varepsilon_{2}=0.25$. We notice that the dip at the band centre that signals the occurrence of a BIC is smeared out if $t$ is finite. Therefore, we are led to the conclusion that the interdot tunneling should be as small as possible to detect to occurrence of BICs in nonequilibrium transport experiments.

\section{Conclusions}

In conclusion, we studied the nonequilibrium transport properties of a DQD system connected in parallel to two leads. Electrons interact with a local vibration mode of each quantum dot. In the absence of interaction, the system supports a BIC, which reveals itself as a Fano antiresonance in the transmission coefficient [21]. When the electron-vibron interaction is taken into account, we found that the main features of the spectral function and the transmission coefficient remain, besides th occurrence of well-defined sidebands. In most cases the transmission coefficient vanishes at the band centre, signaling the occurrence of a BIC. This feature is observed in the differential conductance, which vanishes close to the Fermi level of the source lead if the energy levels of the quantum dots are detuned.

Regarding the experimental validation of the effects of the BICs on the 
nonequilibrium transport in DQD systems, we found that temperature should not exceed the liquid helium temperature for typical values of the parameters. Above this temperature the dip in the differential conductance indicating the occurrence of a BIC is smeared out. We also studied the effects of the interdot tunneling and concluded that it should be small to reveal the BICs. This implies that the dots should be far apart. Another important magnitude to be considered in an experiment is the Huang-Rhys parameter. In our study we set $g=0.25$ and found that electron-vibron interaction does not mask the effect. This value is an order of magnitude larger than the Huang-Rhys parameter found in InP dots of radius $1.2 \mathrm{~nm}$ [35]. Therefore, we are confident that transport experiments in DQD systems could help to understand the nature of the BICs.

Work at Madrid was supported by MINECO (projects MAT2010-17180 and MAT2013-46308). F Domínguez-Adame thanks the Theoretical Physics Group of the University of Warwick for the warm hospitality during the sabbatical leave. P Orellana acknowledges support from FONDECYT (grant 114057), DGIP/USM (internal grant 11.14.68) and CONICYT ACT 1204.

[1] A. W. Holleitner, R. H. Blick, A. K. Hüttel, K. Eberl, J. P. Kotthaus, Science 297 (2002) 70.

[2] A. W. Holleitner, A. Chudnovskiy, D. Pfannkuche, K. Eberl, R. H. Blick, Phys. Rev. B 70 (2004) 075204.

[3] A. Georges, Y. Meir, Phys. Rev. Lett. 82 (1999) 3508.

[4] R. Aguado, D. C. Langreth, Phys. Rev. Lett. 85 (2000) 1946.

[5] R. López, R. Aguado, G. Platero, Phys. Rev. Lett. 89 (2002) 136802.

[6] J. C. Chen, A. M. Chang, M. R. Melloch, Phys. Rev. Lett. 92 (2004) 176801.

[7] A. W. Holleitner, C. R. Decker, H. Qin, K. Eberl, R. H. Blick, Phys. Rev. Lett. 87 (2001) 256802.

[8] M. Sigrist, A. Fuhrer, T. Ihn, K. Ensslin, S. E. Ulloa, W. Wegscheider, M. Bichler, Phys. Rev. Lett. 93 (2004) 066802.

[9] C. Livermore, C. H. Crouch, R. M. Westervelt, K. L. Campman, A. C. Gossard, Science 274 (1996) 1332. 
[10] K. A. Matveev, L. I. Glazman, H. U. Baranger, Phys. Rev. B 54 (1996) 5637.

[11] F. J. Kaiser, S. Kohler, P. Hänggi, M. Malecha, J. Ebbecke, A. Wixforth, H. W. Schumacher, B. Köstner, D. Reuter, A. D. Wieck, J. Phys.: Condens. Matter 20 (2008) 374108.

[12] M. L. Ladrón de Guevara, F. Claro, P. A. Orellana, Phys. Rev. B 67 (2003) 195335.

[13] S. Sasaki, H. Tamura, T. Akazaki, T. Fujisawa, Phys. Rev. Lett. 103 (2009) 266806.

[14] S. Kawaguchi, J. Phys.: Condens. Matter 21 (2009) 395303.

[15] V. Meden, F. Marquardt, Phys. Rev. Lett. 96 (2006) 146801.

[16] C. A. Büsser, F. Heidrich-Meisner, Phys. Rev. Lett. 111 (2013) 246807.

[17] J. von Neumann, E. Wigner, Phys. Z. 30 (1929) 465.

[18] F. Capasso, C. Sirtori, J. Faist, D. L. Sivco, S. Chu, A. Y. Cho, Nature 358 (1992) 565.

[19] Y. Plotnik, O. Peleg, F. Dreisow, M. Heinrich, S. Nolte, A. Szameit, M. Segev, Phys. Rev. Lett. 107 (2011) 183901.

[20] A. Albo, D. Fekete, G. Bahir, Phys. Rev. B 85 (2012) 115307.

[21] C. González-Santander, P. A. Orellana, F. Domínguez-Adame, EPL (Europhysics Letters) 102 (2013) 17012.

[22] U. Fano, Phys. Rev. 124 (1961) 1866.

[23] S. Longhi, G. Della Valle, Sci. Rep. 3 (2013) 2219.

[24] K. Noba, N. Yamada, Y. Uesaka, S. Tanaka, T. Petrosky, J. Phys A: Math. Theor. 47 (2014) 385302.

[25] R. Žitko, J. Mravlje, K. Haule, Phys. Rev. Lett. 108 (2012) 066602.

[26] H. Haug, A. P. Jauho, Quantum Kinetics in Transport and Optics of Semiconductors, Springer, Berlin, 2007. 
[27] T. Holstein, Ann. Phys. 8 (1959) 325.

[28] I. G. Lang, Y. A. Firsov, Sov. Phys. JETP 16 (1963) 1301.

[29] G. D. Mahan, Many-Particle Physics, Kluwer Academic, New York, 2000 .

[30] Z.-Z. Chen, R. Lü, B. Zhu, Phys. Rev. B 71 (2005) 165324.

[31] L. V. Keldysh, Sov. Phys. JETP 20 (1965) 1018.

[32] M. Bagheri Tagani, H. Rahimpour Soleimani, Phys. Scr. 86 (2012) 035706 .

[33] M. Abramowitz, I. A. Stegun, Handbook of Mathematical Functions, Dover Publications, New York, 1972.

[34] J. Song, Q.-f. Sun, J. Gao, X. C. Xie, Phys. Rev. B 75 (2007) 195320.

[35] M. Hamma, R. P. Miranda, M. I. Vasilevskiy, I. Zorkani, J. Phys.: Condens. Matter 19 (2007) 346215. 